\title{
Thermal conductivity of unsaturated clay-rocks
}

\author{
D. Jougnot ${ }^{1,2,3,{ }^{*}}$ and A. Revil ${ }^{1,3}$ \\ ${ }^{1}$ Colorado School of Mines, Green Center, Dept. of Geophysics, Golden, CO 80401, USA \\ ${ }^{2}$ ANDRA, 1-7 rue Jean Monnet, 92298 Chatenay-Malabry, France \\ ${ }^{3}$ CNRS- LGIT (UMR 5559), University of Savoie, Equipe Volcan, Le Bourget-du-Lac, France \\ *now at: Institut of Geophysics, Amphipôle, UNIL, 1015 Lausanne, Switzerland
}

Received: 4 August 2008 - Published in Hydrol. Earth Syst. Sci. Discuss.: 28 August 2008

Revised: 17 December 2009 - Accepted: 22 December 2009 - Published: 18 January 2010

\begin{abstract}
The parameters used to describe the electrical conductivity of a porous material can be used to describe also its thermal conductivity. A new relationship is developed to connect the thermal conductivity of an unsaturated porous material to the thermal conductivity of the different phases of the composite, and two electrical parameters called the first and second Archie's exponents. A good agreement is obtained between the new model and thermal conductivity measurements performed using packs of glass beads and core samples of the Callovo-Oxfordian clay-rocks at different saturations of the water phase. We showed that the three model parameters optimised to fit the new model against experimental data (namely the thermal conductivity of the solid phase and the two Archie's exponents) are consistent with independent estimates. We also observed that the anisotropy of the effective thermal conductivity of the Callovo-Oxfordian clay-rock was mainly due to the anisotropy of the thermal conductivity of the solid phase.
\end{abstract}

\section{Introduction}

The modelling of the thermal conductivity of unsaturated clay-rich materials has applications to the characterization of the thermal anomalies associated with ground water flow in hydrothermal systems (Jardani and Revil, 2009), climate change (Kooi, 2008), and to study the transport properties of deep argillaceous formations under investigation to potentially host nuclear wastes (Giraud et al., 2007) just to cite few applications. In the context of the storage of nuclear waste in deep clay-rock formations, the French Agency for Nuclear Waste Management (ANDRA) has built an underground laboratory in the Callovo-Oxfordian (COx) clay-rock

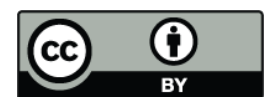

Correspondence to: A. Revil (arevil@mines.edu) formation of the eastern part of the Paris Basin. This laboratory is located at a depth of approximately $500 \mathrm{~m}$. This COx formation seems to provide good characteristics for the HighLevel and Long-Lived (HLLL) nuclear waste disposal (insulation of the radionuclides and toxic chemicals, see ANDRA, 2005a). After the disposal closure, these wastes are expected to produce heat and gases during several decades. The formation temperature increase is expected to be less than $90^{\circ} \mathrm{C}$ (ANDRA, 2005b). The thermal conductivity is therefore a key parameter to assess the performance of the storage facility. The presence of gases (produce by the wastes) or air (due to the gallery ventilation) in the COx formation is also expected to influence the thermal conductivity. Therefore, the understanding of the thermal conductivity behaviour in these two-fluid phase conditions is a very important goal to model the behaviour of such a facility.

Since thermal conductivity is difficult to measure, the present work investigates a relationship between the thermal conductivity to the more easily measured electrical conductivity. The stated aim will be to develop a non-intrusive measurement technique of the electrical conductivity from which the thermal conductivity can then be derived.

\section{Proposed model}

The thermal conductivity is defined by the classical Fourier's law

$\mathrm{Q}=-\lambda \nabla T$,

where $\mathrm{Q}$ (in $\mathrm{W} \mathrm{m}^{-2}$ ) represents the heat flux, $\lambda$ is the thermal conductivity (in $\mathrm{W} \mathrm{m}^{-1} \mathrm{~K}^{-1}$ ), and $T$, the temperature (in $\mathrm{K}$ ). Using an analogy between electrical conductivity and thermal conductivity, Revil (2000) proposed a differential effective medium approach to evaluate the effective thermal conductivity of a "fluid plus solid" mixture. He applied the Bruggeman-Hanai-Sen approach (Sen et al., 1981) to the

Published by Copernicus Publications on behalf of the European Geosciences Union. 
thermal conduction problem. This approach yields for the following formula for the thermal conductivity

$\lambda=\lambda_{f} \phi^{\frac{m}{1-m}}\left(\frac{1-\lambda_{f} / \lambda_{S}}{1-\lambda / \lambda_{S}}\right)$,

where $\phi$ is the porosity, $\lambda_{S}$ and $\lambda_{f}$ are the thermal conductivities of the solid and fluid phases, respectively. Note that this formula needs to be solved iteratively for $\lambda$. The exponent $m$ is the Archie's first exponent (also called the cementation exponent in the description of electrical conductivity of porous rocks). It ranges typically between 1.3 (for unconsolidated sands) to 2.0 (for consolidated rocks) (see Archie, 1942; Lesmes and Fiedman, 2005). The exponent $m$ is defined by the first Archie law $F=\phi^{-m}$ where $F$ is the electrical formation factor (e.g., Archie, 1942; Lesmes and Friedman, 2005). The electrical formation factor is also defined as the ratio between the tortuosity of the pore space $\alpha$ to the connected porosity: $F=\alpha / \phi$ (Revil and Linde, 2006). The parameter $F$ or the exponent $m$ are fundamental textural properties of the porous medium.

The main difference between the thermal and the electrical problem is ratio between solid and fluid conductivities. Indeed, contrary to the electrical conductivities, the thermal conductivity of the solid phase $\lambda_{S}$ is higher than the thermal conductivity of the fluid phase $\lambda_{f}$. This yields to:

$\Theta \equiv \frac{\lambda_{S}}{\lambda_{f}}>1$

Considering Eq. (3), Revil (2000) defined a thermal formation factor given by:

$f=\phi^{\frac{m}{1-m}}$,

in analogy to the electrical formation factor $F$. The two formation factors are related to each other by $f=F^{1 /(m-1)}(\mathrm{Re}-$ vil, 2000). Revil (2000) developed the following closed-form relationship as solution of Eq. (2):

$\lambda=\frac{\lambda_{f}}{f}\left[f \Theta+\frac{1}{2}(1-\Theta)\left(1-\Theta+\sqrt{(1-\Theta)^{2}+4 f \Theta}\right)\right]$.

Note that if the medium is anisotropic, $f$ and $\lambda_{S}$ are second order (symmetric) tensors.

In unsaturated conditions, two fluids (brine, subscript $w$, and air, subscript $a$ ) are present in the pore space of the medium. Following Linde et al. (2006) and Revil et al. (2007) for the conductivity and dielectric constants modelling of unsaturated sandstones, we can relate the thermal conductivity of the fluid phase to the saturations of the brine $s_{w}$ and to the saturation of air $\left(1-s_{w}\right)$ by using a generalized second Archie law:

$\lambda_{f}=s_{w}^{n} \lambda_{w}+\left(1-s_{w}^{n}\right) \lambda_{a}$,

where $\lambda_{w}$ and $\lambda_{a}$ are the thermal conductivities of the brine and air, respectively, $n$ is the second Archie exponent ( $n \approx 2.0 \pm 0.5$, Archie, 1942). This exponent describes the evolution of the water phase tortuosity $\alpha_{w}(\geq \alpha)$ with the saturation $s_{w}$. Equation (6) appears as a "generalized" Archie's law using the thermal conductivities of the two fluid phases and their relative volumetric fractions. Typically, $\lambda_{a}=0.0255 \mathrm{~W} \mathrm{~K}^{-1} \mathrm{~m}^{-1}$ and $\lambda_{w}=0.5984 \mathrm{~W} \mathrm{~K}^{-1} \mathrm{~m}^{-1}$ (Clauser and Huenges, 1995). In the case of the electrical conductivity, the conductivity of air is zero. The first Archie exponent $m$ and the electrical formation factor $F=\phi^{-m}$ are fundamental textural properties of the medium. Therefore, they should not be dependent upon the saturation of the water phase (e.g., Yaramanci et al., 2002; Kruschwitz and Yaramanci, 2004; Linde et al., 2006). Combining Eqs. (6) and (3) into Eq. (5) provides a new model describing the thermal conductivity as a function of the thermal conductivity of the different phases, and two electrical parameters.

\section{Application to glass beads}

In the previous section we developed a model to determine the effective thermal conductivity of partially saturated media from electrical properties (Eqs. 3 to 6). In order to test the validity of this model, we compared its predictions to experimental data from the literature. In our approach, the model parameters were optimised according to measurements using the Simplex algorithm. The cost function to minimize was the sum of square residuals $\left(S S_{R}\right)$ between the $N$ experimentally observed data $y_{i}$ and the $N$ corresponding calculated one $y_{i}^{\prime}$ :

$S S_{R}=\sum_{i=1}^{N}\left(y_{i}-y_{i}^{\prime}\right)^{2}$

We used a normalised sum of square residuals $R_{N}=S S_{R} / N$ to compare experiments with different numbers of measurements.

The data from Kohout et al. (2004) were obtained using a pack of glass beads. The mean diameter of the beads is $d=200 \mu \mathrm{m}$ and the porosity is $\phi=0.39$. The thermal conductivities of the glass beads, the water phase, and the air phase are $\lambda_{S}=0.80 \mathrm{~W} \mathrm{~K}^{-1} \mathrm{~m}^{-1}, \lambda_{w}=0.61 \mathrm{~W} \mathrm{~K}^{-1} \mathrm{~m}^{-1}$, and $\lambda_{a}=0.02 \mathrm{~W} \mathrm{~K}^{-1} \mathrm{~m}^{-1}$, respectively (from Kohout el al., 2004). Therefore, only two parameters had to be optimized for the model-data comparison: $m$ and $n$, the two Archie's exponents. Figure 1 shows that our model is able to describe fairly well the behaviour of the thermal conductivity with respect to the saturation of the water phase. The best fit was obtained with $m=1.7 \pm 0.1$ and $n=1.5 \pm 0.1$, which yields $R_{N}=4.4 \times 10^{-3}$ (Fig. 1). The value of $m$ agrees with the values of $m=1.7$ determined numerically by Schwartz and Kimminau (1987) and usually the value of $n$ is relatively close to the value of $m$. These results seem to validate our model to determine unsaturated thermal conductivity from electrical measurements in unconsolidated porous medium. 
Table 1. Vertical limits and average porosities of geological units in the COx clay-rocks (ANDRA, 2009).

\begin{tabular}{lcccc}
\hline Geological unit & Top $(\mathrm{m})^{\mathrm{a}}$ & Bottom $(\mathrm{m})^{\mathrm{a}}$ & \multicolumn{2}{c}{$\begin{array}{l}\text { Porosity }^{\mathrm{b}} \\
\text { Standard deviation (-) }\end{array}$} \\
\hline $\mathrm{C} 2 \mathrm{~d}$ & 417 & 437 & 0.137 & 0.047 \\
$\mathrm{C} 2 \mathrm{c}$ & 437 & 456 & 0.163 & 0.048 \\
$\mathrm{C} 2 \mathrm{~b} 2$ & 456 & 472 & 0.178 & 0.019 \\
$\mathrm{C} 2 \mathrm{~b} 1$ & 472 & 508 & 0.166 & 0.022 \\
$\mathrm{C} 2 \mathrm{a}$ & 508 & 554 & 0.157 & 0.043 \\
$\mathrm{COx}^{\mathrm{c}}$ & 417 & 554 & $0.160^{\mathrm{c}}$ & $0.030^{\mathrm{c}}$ \\
\hline
\end{tabular}

a Vertical limits in the EST205 borehole (Gaucher et al., 2004)

b Calculated from the gravimetric water content with $\rho_{\mathrm{W}}=1000 \mathrm{~kg} \mathrm{~m}^{-3}$ and $\rho_{\mathrm{g}}=2690 \mathrm{~kg} \mathrm{~m}^{-3}$

${ }^{\mathrm{c}}$ Mean values of the Callovo-Oxfordian formation

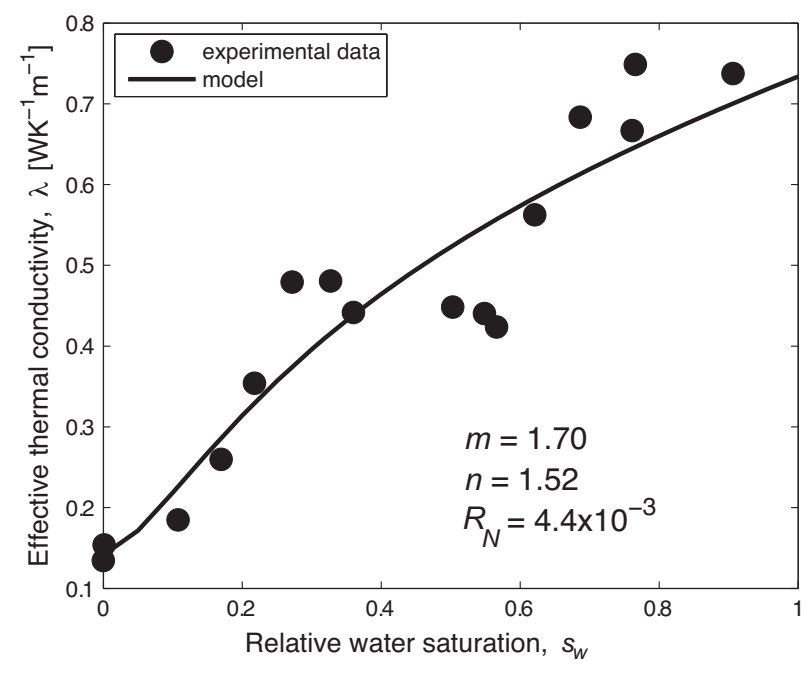

Fig. 1. Fitting proposed model to experimental thermal conductivity measurements of randomly compacted glass beads (data from Kohout et al., 2004).

\section{Application to a clay-rock}

We compare now our model to the clay-rocks effective thermal conductivities measured at various relative water saturations in the range 0 (dry) to 1 (saturated) by Homand (1998). The core samples used for these measurements were collected at Bure (Meuse/Haute-Marne, France) in the CallovoOxfordian (COx) formation at a depth interval ranging from 450 to $525 \mathrm{~m}$. This formation is presently investigated by ANDRA to be a potential host for high-level long-lived nuclear waste because of their low permeability $\left(\sim 10^{-20} \mathrm{~m}^{2}\right)$ and high specific surface area (ANDRA, 2005a). The COx formation has been the target of numerous scientific investigations for the last two decades and an underground research laboratory has recently been built by ANDRA to have a direct access to the formation and to conduct in situ experimentation.
The COx clay-rocks can be conceptualized by a clay matrix (illite and interstratified illite/smectite) with grain inclusions (meanly quartz and carbonates). The different geological units to describing the COx formation are the $\mathrm{C} 2 \mathrm{~d}, \mathrm{C} 2 \mathrm{c}$, C2b, and C2a units (Gaucher et al., 2004). The following limits are from Gaucher et al. (2004) for the EST205 borehole but the mineralogical variations are progressive. The upper part of the formation (unit $\mathrm{C} 2 \mathrm{~d}$, roughly from 417 to $437 \mathrm{~m}$ deep at the position of the underground laboratory) is composed of interbedded black bioturbated clay-rock and slightly bioturbated calcareous clay-rock. This unit presents important lateral variation in the mineralogical composition. The carbonate fraction varies between 80 and $40 \%$ while the clay fraction does from 15 to $45 \%$. Below $437 \mathrm{~m}$ the COx becomes more homogeneous with a clay fraction of 40 $50 \%, 20-35 \%$ of carbonates, and $25-35 \%$ of silica (units $\mathrm{C} 2 \mathrm{c}$ and $\mathrm{C} 2 \mathrm{~b}$ ). The $\mathrm{C} 2 \mathrm{c}$ unit is composed of interbedded black silty-calcareous clay-rock and black marl. The $\mathrm{C} 2 \mathrm{~b}$ unit is usually decomposed in two subunits: $\mathrm{C} 2 \mathrm{~b} 2$, black siltycalcareous clay-rock (456-472 m), and C2b1, black bioclastic and pyritic silty-calcareous clay-rock $(472-508 \mathrm{~m})$. The $\mathrm{C} 2 \mathrm{~b} 1$ unit contains the Maximum Clay Zone (MCZ) between 486 and $489 \mathrm{~m}$ (the clay fraction peak reaches approximatively $60 \%$ ). The main galleries of the underground laboratory were built at a depth of approximately $490 \mathrm{~m}$, just below the MCZ. The C2a unit is located below approximately $508 \mathrm{~m}$ and is composed by a silty clay-rock. Note that from the MCZ to the bottom of the COx, the clay fraction contains kaolinite. Table 1 presents the vertical limit of these units in the EST205 borehole (above the laboratory and few tens meters from EST104) and the mean measured porosities and standard deviations for each units.

ANDRA (2005a) studied the total porosity distribution in the $\mathrm{C} 2 \mathrm{~b}$ unit and propose conceptual model for the pore diameter distribution relatively to the volume (Fig. 2). Three scales of porosity are proposed: (i) a microporosity with a pore diameter $<2 \mathrm{~nm}$, (ii) a mesoporosity with a pore diameter comprised between 2 and $50 \mathrm{~nm}$, and (iii) a macroporosity 
with a pore diameter $>50 \mathrm{~nm}$. From these studies, ANDRA (2005a) notice that a part of the porosity is filled with bound water: fraction of the mesoporosity and all the microporosity (corresponding to a pore scale $<10 \mathrm{~nm}$ ). ANDRA (2005a) considers a total porosity reference $\phi_{T}=0.18$ in the $\mathrm{C} 2 \mathrm{~b}$ unit and a bound water porosity $\phi_{B}=0.07$ (therefore $\phi_{\mathrm{B}}=0.39 \phi_{T}$ ) (see Fig. 2).

These vertical variations in the mineralogical composition and porosities impact the transport properties of the clayrocks. Therefore the different units were tested by ANDRA to assess their transport properties. Homand (1998) used seven clay-rocks core samples extracted from the EST104 borehole at different depths between 450 and $525 \mathrm{~m}$ (in units $\mathrm{C} 2 \mathrm{c}, \mathrm{C} 2 \mathrm{~b} 2, \mathrm{C} 2 \mathrm{~b} 1$, and $\mathrm{C} 2 \mathrm{a}$ ). The collected core samples were re-sampled in three orientations relatively to the stratification: parallel, perpendicular and with a $45^{\circ}$ angle (21 samples: 3 samples per cell). The final samples used for the measurements were small cylinders with a $36.8 \mathrm{~mm}$ diameter, a $10.5 \mathrm{~mm}$ length and a $28 \pm 1 \mathrm{~g}$ mean mass. For each sample, mineralogy, density and porosity were determined using the left-other from re-sampling. Different methods were used to probe the porosity of these core samples and provide complementary information to each other. Homand (1998) used two methods: Mercury intrusion experiments (Washburn, 1921) and porosity determination by the triple weight measurements. From ANDRA (2005a), we know that mercury intrusion is unable to investigate pore size below $4 \mathrm{~nm}$ and therefore provides only a lower bound for the total porosity. In contrast, calculations based on volumetric weights with a pycnometer provide an upper bound on the total porosity. We have decided to use below this calculated porosity because it is likely to be close to the real porosity.

The thermal conductivities of the samples were measured by Homand (1998) using a commercial flash diffusivimeter built by CEDIP (under an INSA Lyon license). A heat flash (600 to $1000 \mathrm{~J}$ during 1 to $10 \mathrm{~ms}$ ) is created on one side of the sample and the temperature variation is measured on the other side (Fig. 3). The evolution of the temperature difference between the sensor and the isothermal bloc gave the thermal diffusivity. Then, Homand (1998) measured the specific heat of the sample using a calorimeter. The thermal conductivity was obtained from the value of the thermal diffusivity and the specific heat of each core sample. This procedure was applied to each core sample at different water saturations.

The measurements were performed during a resaturation process. First, each sample was dried in a $105^{\circ} \mathrm{C}$ oven during $48 \mathrm{~h}$ to measure the dry mass. Then, they were placed in a dessicator (confined atmosphere) with a controlled relative humidity in order to manage the re-saturation. This relative humidity was controlled using saline solutions in equilibrium with the dessicator confined atmosphere. Six solutions were used to fix relative humidity (from 66 to $98 \%$ ) and then to re-saturate the samples following six steps in saturation. After trial and error tests, Homand (1998) left the samples in

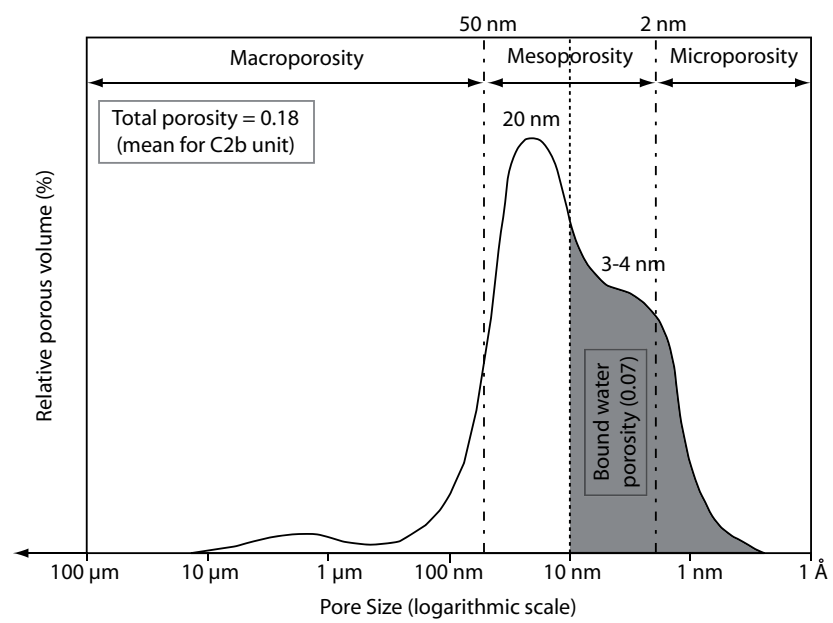

Fig. 2. Conceptual model for pore size distribution in the $\mathrm{C} 2 \mathrm{~b}$ unit of Callovo-Oxfordian clay-rock (ANDRA, 2005a).

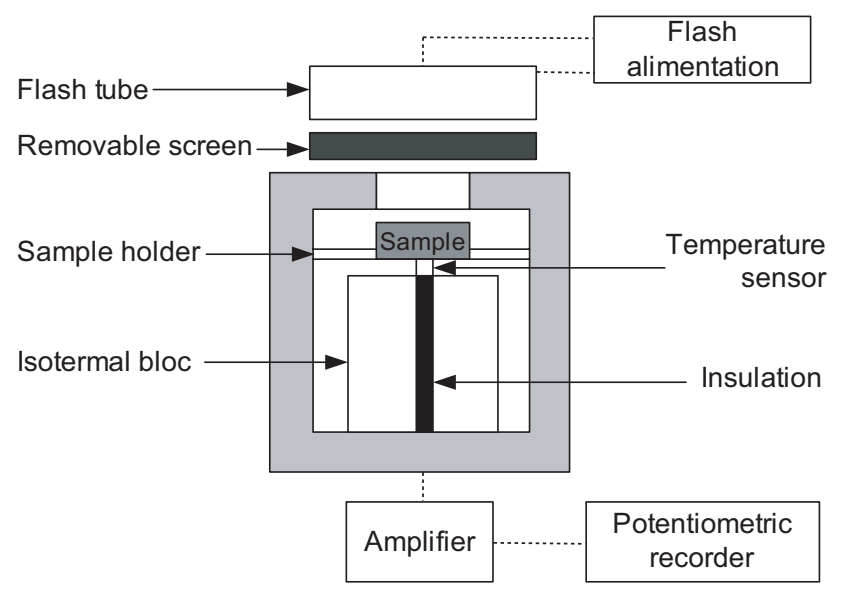

Fig. 3. CEDIP device to measure the thermal diffusivity of clayrock samples (Homand, 1998).

the dessicator during three weeks for each saturation step to reach an equilibrium between the fixed relative humidity of the atmosphere and the sample.

In order to test our model against the measurements performed by Homand (1998), we optimized three model parameters per sample: $m, n$, and $\lambda_{S}$. The solid phase being composed by several minerals with different thermal conductivities, the equivalent grain thermal conductivity is unknown. It can be computed from classical mixing law or homogenization (see Gruescu et al., 2007; Giraud et al., 2007). In the present work, we choose to optimize directly $\lambda_{S}$ to limit the uncertainties due to additional computations. The thermal formation factor, $f$, is computed from Eq. (4) using porosities calculated by Homand (1998) from triple weight measurements. The values of the water conductivities of air and water were taken from Clauser and Huenges (1995) (see above in Sect. 2). The optimization was performed 

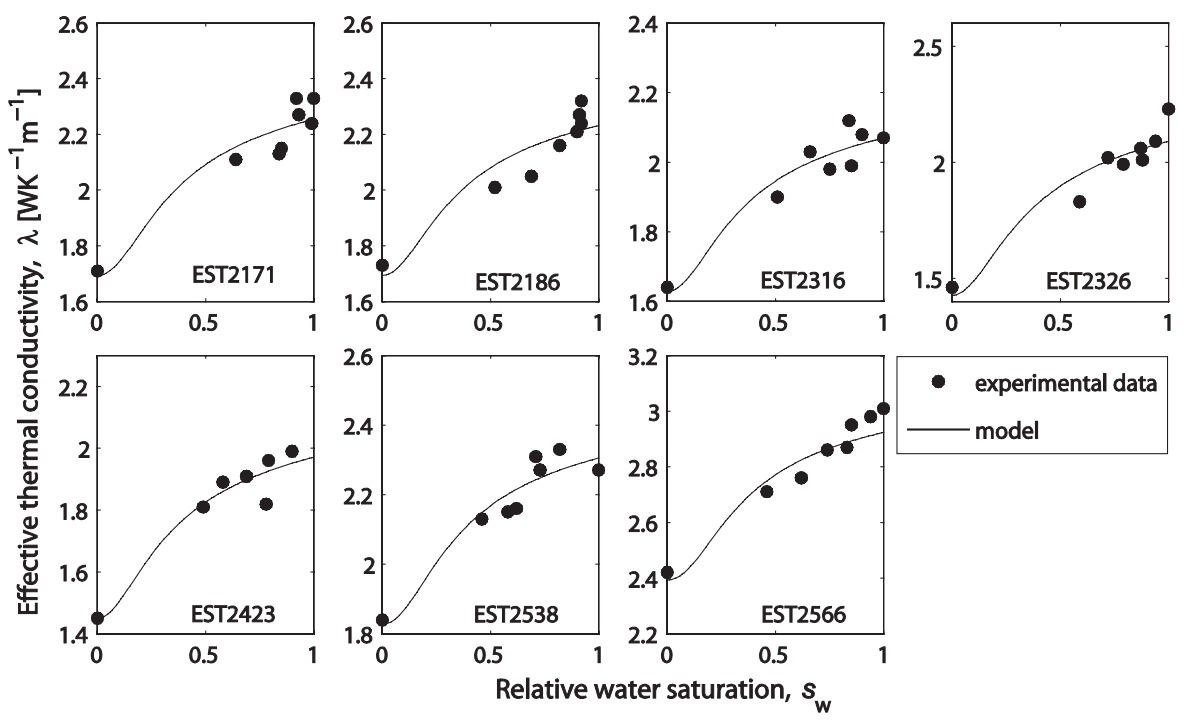

Fig. 4. Optimized model and experimental data comparison for seven samples of COx clay-rocks. Optimized parameter values are located in Table 2.

Table 2. Optimized parameters for seven COx clay-rock samples (porosity corresponding to the calculated porosity from Homand, 1998).

\begin{tabular}{lcccccc}
\hline Sample & $\begin{array}{c}\text { Geological } \\
\text { unit }\end{array}$ & $\begin{array}{c}\phi \\
(-)\end{array}$ & $\begin{array}{c}m \\
(-)\end{array}$ & $\begin{array}{c}N \\
(-)\end{array}$ & $\begin{array}{c}\lambda_{S} \\
\left(\mathrm{~W} \mathrm{~K}^{-1} \mathrm{~m}^{-1}\right)\end{array}$ & $\begin{array}{c}R_{N} \\
(-)\end{array}$ \\
\hline EST 2171 & C2c & 0.13 & $1.45 \pm 0.01$ & $2.09 \pm 0.52$ & $2.38 \pm 0.01$ & $3.8 \times 10^{-3}$ \\
EST 2186 & C2c & 0.16 & $1.37 \pm 0.06$ & $2.04 \pm 0.51$ & $2.35 \pm 0.06$ & $4.3 \times 10^{-3}$ \\
EST 2316 & C2b2 & 0.15 & $1.37 \pm 0.01$ & $2.05 \pm 0.46$ & $2.16 \pm 0.06$ & $1.9 \times 10^{-3}$ \\
EST 2326 & C2b1 & 0.17 & $1.42 \pm 0.01$ & $2.02 \pm 0.49$ & $2.24 \pm 0.01$ & $4.9 \times 10^{-3}$ \\
EST 2423 & C2b1 & 0.17 & $1.38 \pm 0.01$ & $1.99 \pm 0.35$ & $2.08 \pm 0.02$ & $2.1 \times 10^{-3}$ \\
EST 2538 & C2b1 & 0.08 & $1.54 \pm 0.09$ & $2.11 \pm 0.52$ & $2.41 \pm 0.01$ & $2.0 \times 10^{-3}$ \\
EST 2566 & C2a & 0.11 & $1.42 \pm 0.39$ & $2.16 \pm 0.53$ & $3.04 \pm 0.25$ & $2.8 \times 10^{-3}$ \\
\hline
\end{tabular}

by combining the Simplex algorithm with a Monte Carlo method used to generate 100 prior values of each model parameter. From the final values returned by the Simplex algorithm for each model parameter, we estimated the median value and the standard deviation.

The test conducted by Homand (1998) showed, as expected, a decrease of the thermal conductivities with desaturation. Note that there is no intermediate saturations investigated in the range $0<s_{w}<0.4$ and that this range corresponds to the bound water porosity $\phi_{B}$. This bound water porosity is the fraction of the total connected porosity occupied by the hydration water of the mineral surface. Homand (1998) demonstrated the anisotropic character of the thermal conductivity of the COx clay-rock by performing measurements along the axes relative to the stratification (parallel, perpendicular, and at $45^{\circ}$ ). We first discuss below the measurements parallel to the stratification.
The prior value of $m$ was provided by Revil et al. (2005) and is equal to $m=1.95 \pm 0.04$ for undisturbed water-saturated COx clay-rock. Leroy and Revil (2009) found $m=2.2$ for the Mancos shale (an early cretaceous carbonaceous clay rock from eastern Utah). Using tracer diffusion experiments, Jougnot et al. (2009) found $m=2.0$ for the COx clay-rocks in saturated conditions. If the clay material contains microcracks because of desiccation at high temperatures, the value of $m$ is expected to be much lower than 2.0, typically in the range 1.3-1.5.

The values of the optimized model parameters were reported in Table 2. The overall fit to the experimental data were excellent. Indeed the mean of $R_{N}$ for the 7 samples is $3.1 \times 10^{-3}$ (see Fig. 4). The range of the optimized parameters is very narrow (Table 1$): m \in(1.37 ; 1.54)$, $n \in(1.99 ; 2.16)$, and $\lambda_{S} \in(2.08 ; 3.04) \mathrm{W} \mathrm{K}^{-1} \mathrm{~m}^{-1}$. We note that the values of $m$ are quite low in regards to the value reported by the works on saturated undisturbed clay-rocks 
(see above). However, these values are similar to the ones obtained by Kruschwitz and Yaramancy (2004) using spectral induced polarization measurements on a similar clayrock with microcracks. They performed these electrical measurements on Opalinus clay (from Mont Terry, Switzerland) at different saturations degree after a prior drying $\left(40^{\circ} \mathrm{C}\right.$ until the weight stabilization) and complete rewetting $(\mathrm{Kr}$ uschwitz, 2009). They obtained $1.3<m<1.5$ and $1.5<n$ $<2.0$, by fitting an Archie's law (Schopper, 1982).

We want to notice here that the heating treatment of Homand (1998) required to get away the water phase $\left(105^{\circ} \mathrm{C}\right.$ during $48 \mathrm{~h}$ ) might have damaged the structure of the core samples by creating microcracks. Such structural modifications were observed in similar conditions by Montes et al. (2004) at $50^{\circ} \mathrm{C}$ using an environmental scanning electron microscope. This study also showed that a part of these modifications are irreversible, thus the COx clay-rocks never entirely recover their undisturbed properties even after a complete rewetting. Ghorbani et al. (2009) observed similar change in the microstructure for the COx clay-rock using spectral induced polarization measurements after exposing the core samples to different temperatures ranging from 70 to $105^{\circ} \mathrm{C}$.

Our assumption is that the presence of microcracks due to the heating phase and the dessication is responsible for a decrease of the value of $m$ with respect to its nominal value for an undisturbed material. Indeed, as we explained in the previous section, $m$ is related to the tortuosity of the porous material and $m$ increase with the tortuosity: $m=1.3$ for a sand and $m>2$ for a consolidated rock. As microcracks provide shortcut to the fluid path, the tortuosity of the pore space decreases. Thus, the presence of microcracks formation explained the range of values of $m$.

In order to test further our model on these data parallel to the stratification, we performed two additional tests (Fig. 5). First, we fixed $m$ by taking the value of Revil et al. (2005) $(m=1.95)$ and we optimized only $n$ and $\lambda_{S}$ with the Simplex combined with a Monte-Carlo method (Test 2 in Fig. 5). We observed that $R_{\mathrm{N}}$ increased by 2 orders of magnitude. The range of the values of $n$ became wider $(n \in(1.57 ; 2.26))$ and $\lambda_{S}$ increased $\left(\lambda_{S} \in(2.78 ; 3.96) \mathrm{W} \mathrm{K}^{-1} \mathrm{~m}^{-1}\right)$. The choice of $m=1.95$ is therefore not a good choice for the damaged clay-rock samples. Then, we performed a second test by taking $n=2$ and we optimized $m$ and $\lambda_{S}$ (Test 3 in Fig. 5). We observed no important changes between these results and those obtained above by optimizing the three parameters $m$, $n$, and $\lambda_{S}$ (the mean of $R_{\mathrm{N}}$ is $3.2 \times 10^{-3}, m \in(1.37 ; 1.54)$ and $\left.\lambda_{S} \in(2.08 ; 3.07) \mathrm{W} \mathrm{K}^{-1} \mathrm{~m}^{-1}\right)$. Therefore, the value of $n=2$ is a good default value. This value agrees with an independent work performed by Jougnot et al. (2010) using electrical resistivity measurements at various saturations (in the range 0.15-0.80) of undisturbed core samples from the COx formation. They found $n$ in the range 1.8 to 2.0 .

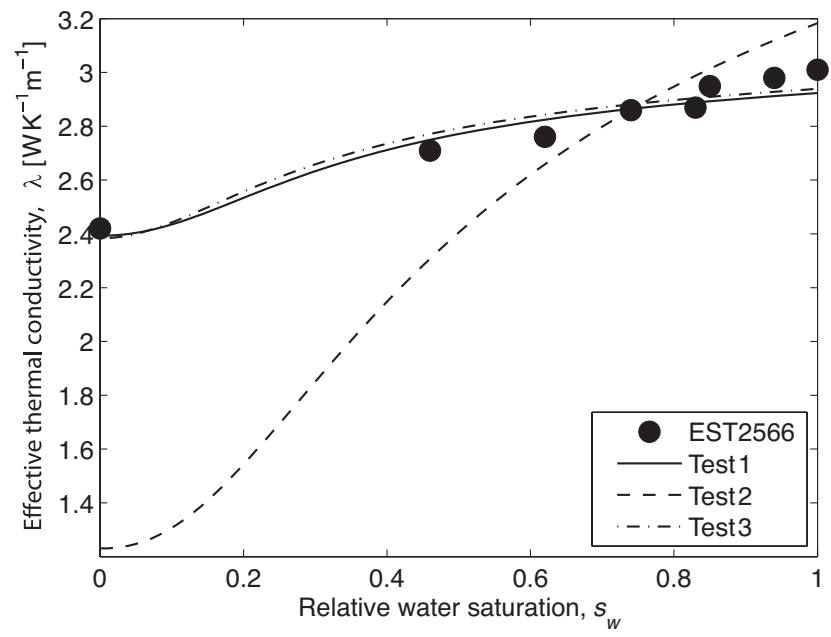

Fig. 5. Three tests on the optimization procedure. Test 1 corresponds to the regular three parameters optimization $\left(m, n, \lambda_{S}\right)$. Test 2 and 3 correspond to the two parameters optimization: with $m=1.95$ and $n=2$ fixed, respectively.

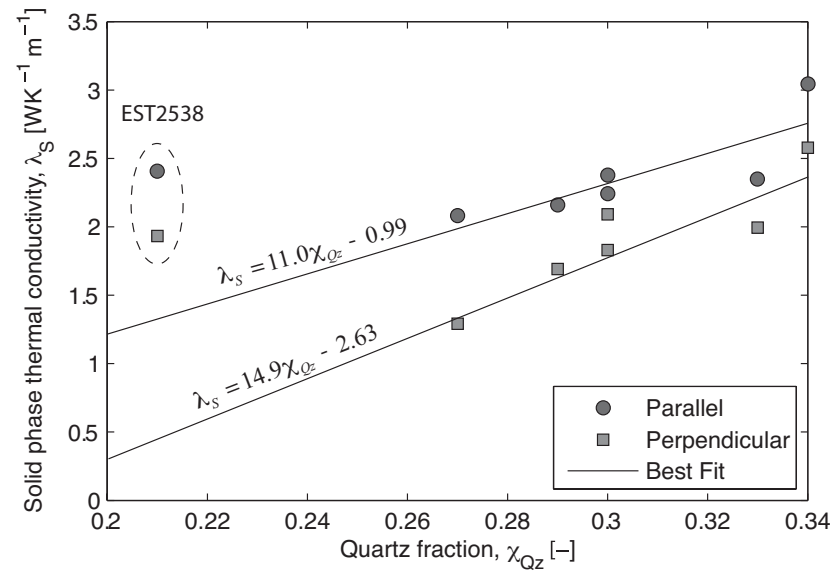

Fig. 6. Quartz fraction $\left(\chi_{Q \mathrm{Q}}\right)$ versus optimized solid phase thermal conductivity. Dark circles and grey squares correspond to parallel and perpendicular to stratification $\lambda_{S}$, respectively. Note that the EST2538 sample does not follow the other values trend.

We also compared the optimized values of the thermal conductivity of the solid phase from estimate using the mineral composition of the core samples (from Homand, 1998). We observed a quite good correlation between the quartz fraction $\chi_{\mathrm{Qz}}$ and the thermal conductivity of the solid phase: $\lambda_{S}=11.0 \chi_{\mathrm{Qz}}-0.99\left(R^{2}=0.68\right.$, excluding EST2538, Fig. 6). We tried several classical averaging methods to determine $\lambda_{S}$ from the mineralogy but none was better than the previous fit. We also fitted the optimized $\lambda_{S}$ for measurements perpendicular to the stratification. We obtained $\lambda_{S}=14.9 \chi_{\mathrm{Qz}}-2.63$ $\left(R^{2}=0.81\right.$, excluding EST2538). 


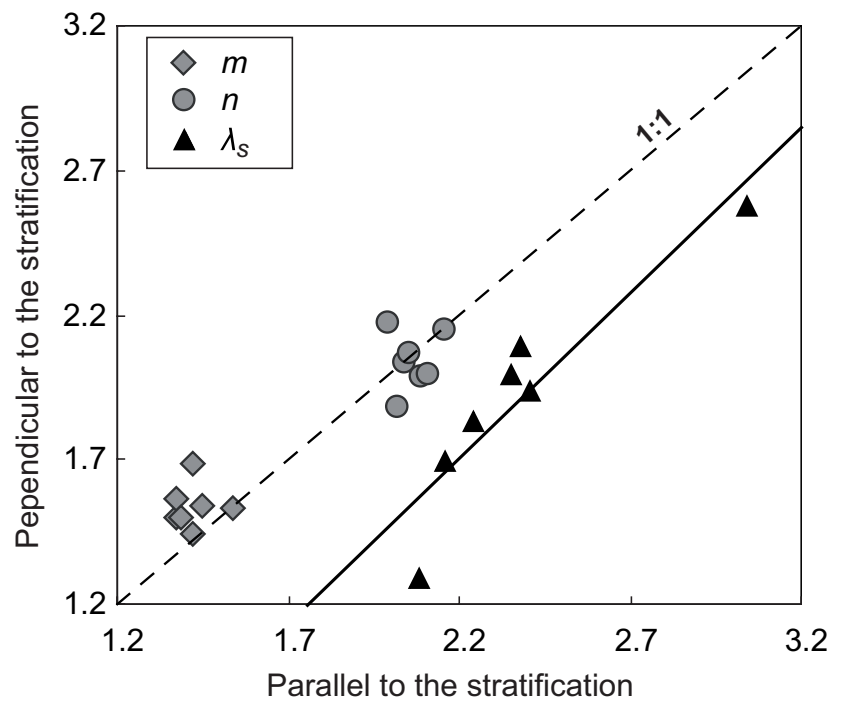

Fig. 7. Comparison between optimized parameters $\left(m, n\right.$, and $\left.\lambda_{S}\right)$ with respect of the COx clay-rock bedding. The plain line presents a linear regression between the two solid phase thermal conductivity optimized from measurements made parallel and perpendicular to the stratification $\left(\lambda_{S(/ /)}\right.$ and $\lambda_{S(+)}$, respectively, with $\lambda_{S(/ /)}=1.14$ $\left.\lambda_{S(+)}-0.81, R_{\mathrm{N}}=0.020\right)$.

We also investigated the data reported by Homand (1998) for the direction perpendicular to the stratification (not shown here). In order to quantify the anisotropy of the clay-rock, we estimate the following mean ratio for the seven samples: $m_{(/ /)} / m_{(+)}=0.926 \pm 0.115, n_{(/ /)} / n_{(+)}=1.015 \pm 0.106$, and $\lambda_{S(/ /)} / \lambda_{S(+)}=1.265 \pm 0.320$ where the subscripts $(/ /)$ and (+) corresponds to the parameter inverted for the data parallel and perpendicular with respect to the stratification, respectively. We observed no strong anisotropy for $n$, a small anisotropy for $m$, but clearly a strong anisotropy for $\lambda_{S}$ (see Fig. 7). The anisotropy of the solid phase is due to the fabric of the grains with preferential orientations in the plane of stratification. Therefore this preferential orientation dictates the anisotropy of the porous material in this case.

\section{Conclusions}

In the present work, we have extended the thermal conductivity model proposed by Revil (2000) to unsaturated conditions. The advantage of this model is to connect the effective thermal conductivity to the electrical Archie's exponents: $m$ and $n$. The present work achieved the goal to reproduce fairly well the behaviour of the thermal conductivity with respect to the saturation using a differential effective medium approach and a generalized Archie's law. The value of $n=2$ can be used to interpret electrical resistivity data (or georadar data) of damaged clay-rocks in terms of thermal conductivity distribution. We may suppose that in undisturbed COx clay- rocks $m=2(\sim 1.95)$ could be used and that $m<2$ would correspond to a fingerprint of the presence of microcracks due to desiccation. Using this approach, it seems possible to image non-intrusively the thermal conductivity of the formation using electrical methods. This will be the purpose of a future work to perform such an in situ test.

Acknowledgements. This work is supported by the CNRS, the French Agency for Radioactive Waste Management (ANDRA) (S. Altmann and D. Coelho) and GDR-FORPRO (J. Lancelot). The $\mathrm{PhD}$ Thesis of D. Jougnot is supported by ANDRA. D. Jougnot thanks D. Coelho, P. Leroy, and J-C. Robinet for the fruitful discussions. A. Revil strongly thanks T. Young for his support at CSM. We thank the two anonymous referees and the associate editor Gerrit H. de Rooij for their very constructive comments.

Edited by: G. H. de Rooij

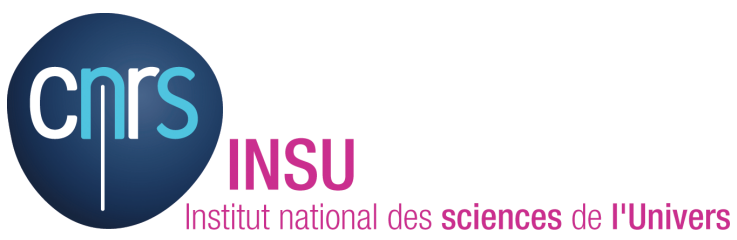

The publication of this article is financed by CNRS-INSU.

\section{References}

ANDRA: Dossier 2005 Argile-Référentiel du Site de Meuse/HauteMarne, Rep. C.RP.ADS.04.0022, June 2005, ANDRA, Châtenay-Malabry, France, 2005a.

ANDRA: Dossier 2005 Argile-Tome-Evolution phénoménologique du stockage géologique, Internal report ANDRA, 2005b.

ANDRA: Dossier 2009 Argile, Referentiel du site de Meuse HauteMarne, Internal report, 2009.

Archie, G. E.: The electrical resistivity log as an aid in determining some reservoir characteristics, T. Am. I. Min. Met. Eng., 146, 54-62, 1942.

Clauser, C. and E. Huenges: Thermal conductivity of rocks and minerals, in: Rocks Physics and Phase Relations: a handbook of physical constants, AGU Reference Shelf 3, edited by: Ahrens, T., American Geophysical Union, Washington, DC, USA, 105126, 1995.

Gaucher, E., Robelin, C., Matray, J. M., Negrel, G., Gros, Y., Heitz, J. F., Vinsot, A., Rebours, H., Cassabagnere, A., and Bouchet, A.: ANDRA underground research laboratory: interpretation of the mineralogical and geochemical data acquired in the Callovo-Oxfordian Formation by investigative drilling, Phys. Chem. Earth, 29, 55-77, doi:10.1016/j.pce.2003.11.006, 2004.

Ghorbani, A., Cosenza, Ph., Revil, A., Zamora, M., Schmutz, M., Florsch, N., and Jougnot, D.: Non-invasive monitoring of water content and textural changes in clay-rocks using spectral induced polarization: A laboratory investigation, Appl. Clay Sci., 43, 493-502, doi:10.1016/j.clay.2008.12.007, 2009.

Giraud A., Gruescu, C., Do, D. P., Homand, F., and Kondo, D.: Effective thermal conductivity of transversely isotropic media with arbitrary oriented ellipsoïdal inhomogeneities, Int. J. Solids Struct., 44(9), 2627-2647, 2007. 
Giroux, B. and Chouteau, M.: A hydrogeophysical synthetic model generator, Comput. Geosci., 34(9), 1080-1092, 2008.

Gruescu, I. C., Giraud, A., Homand, F., Kondo, D., and Do, D. P.: Effective thermal conductivity of partially saturated rocks, Int. J. Solids Struct., 44, 811-833, doi:10.1016/j.ijsolstr, 2007.

Homand, F.: Mesures thermiques sur le site est, Technical Report ANDRA, DRP0ENG 98-009/A, INPL-LAEGO, France, 1998.

Jardani, A. and A. Revil: Stochastic joint inversion of temperature and self-potential data, Geophys. J. Int., 179(1), 640-654, doi:10.1111/j.1365-246X.2009.04295.x, 2009.

Jougnot, D., Revil, A., and Leroy, P.: Diffusion of ionic tracers in the Callovo-Oxfordian clay-rock using the Donnan equilibrium model and the formation factor, Geochim. Cosmochim. Acta, 73, 2712-2726, 2009.

Jougnot, D., Ghorbani, A., Revil, A., Leroy, P., and Cosenza, P.: Spectral Induced Polarization of partially saturated clayrocks: A mechanistic approach, Geophys. J. Int., 180, 210-224, doi:10.1111/j.1365-246X.2009.04426.x, 2010.

Kohout, M., Collier, A. P., and Štepánek, F.: Effective thermal conductivity of wet particle assemblies, Int. J. Heat Mass Tran., 47, 5565-5574, 2004.

Kooi, H.: Spatial variability in subsurface warming over the last three decades; insight from repeated borehole temperature measurements in the Netherlands, Earth Planet. Sc. Lett., 270(1-2), 86-94, 2008.

Kruschwitz S. and Yaramanci, U.: Detection and characterization of the disturbed rock zone in claystone with complex resistivity method, J. Appl. Geophys., 57, 63-79, doi:10.1016/j.jappgeo.2004.09.003, 2004.

Kruschwitz S., personal communication, 2009.

Leroy, P. and Revil, A.: Spectral induced polarization of clays and clay-rocks, J. Geophys. Res., 114, B10202, doi:10.1029/2008JB006114, 2009.

Lesmes, D. P. and Friedman, S.: Relationships between the electrical and hydrogeological properties of rocks and soils, in: Hydrogeophysics, edited by: Rubin, Y. and Hubbard, S., Springer, New York, USA, 87-128, 2005.
Linde, N., Binley, A., Tryggvason, A., Pedersen, L. B., and Revil, A.: Improved hydrogeophysical characterization using joint inversion of cross-hole electrical resistance and ground penetrating radar traveltime data, Water Resour. Res., 42, W12404, doi:10.1029/2006WR005131, 2006.

Montes H. G., Duplay, J., Martinez, L., Escoffier, S., and Rousset, D.: Structural modifications of Callovo-Oxfordian argillite under hydration/dehydration conditions, Appl. Clay Sci., 25, 187-194, 2004.

Revil, A.: Thermal conductivity of unconsolidated sediments with geophysical applications, J. Geophys. Res., 105(B7), 16 749-16 768, 2000

Revil, A., Leroy, P., and Titov, K.: Characterization of transport properties of argillaceous sediments. Application to the Callovo-Oxfordian Argillite, J. Geophys. Res., 110, B06202, doi:10.1029/2004JB003442, 2005.

Revil, A. and Linde, N.: Chemico-electromechanical coupling in microporous media, J. Colloid Interface Sci., 302, 682-694, doi:10.1016/j.jcis.2006.06.051, 2006.

Revil, A., Linde, N., Cerepi, A., Jougnot, D., Matthäi, S., and Finsterle, S.: Electrokinetic coupling in unsaturated porous media, J. Colloid Interf. Sci., 313, 315-327, doi:10.1016/j.jcis.2007.03.037, 2007.

Schopper, J. R.: Electrical conductivity of rocks containing electrolytes, in: Landolt-Brnstein Numerical Data and Functional Relationships in Science and Technology, Group V, Physical Properties of Rocks, edited by: Hellwege, K.-H., vol. 1b, Springer-Verlag, Heidelberg, New York, 276-291, 1982.

Schwartz, L.M. and Kimminau, S.: Analysis of electrical conduction in the grain consolidated model, Geophysics, 52, 1402 $1411,1987$.

Sen, P. N., Scala, C., and Cohen, M. H.: A self-similar model for sedimentary rocks withapplication to the dielectric constant of fused glass beads, Geophysics, 46, 781-795, 1981.

Washburn, E. W.: Note on a Method of Determining the Distribution of Pore Sizes in a Porous Material, P. Natl. Acad0. Sci. USA, 7(4), 115-116, 1921.

Yaramanci, U., Lange, G., and Hertrich, M.: Aquifer characterisation using Surface NMR jointly with other geophysical techniques at the Nauen/Berlin test site, J. Appl. Geophys., 50, 4765, doi:10.1016/S0926-9851(02)00129-5, 2002. 